(อ) OPEN ACCESS

\title{
First pass effect with contact aspiration and stent retrievers in the Aspiration versus Stent Retriever (ASTER) trial
}

\author{
Célina Ducroux, ${ }^{1}$ Michel Piotin, ${ }^{1}$ Benjamin Gory, ${ }^{2}$ Julien Labreuche, ${ }^{3}$ Raphael Blanc, \\ Malek Ben Maacha, ${ }^{1}$ Bertrand Lapergue, ${ }^{4}$ Robert Fahed (D) , ${ }^{1}$ for the ASTER Trial \\ investigators
}

\begin{abstract}
- Additional material is published online only. To view please visit the journal online (http://dx.doi.org/10.1136/ neurintsurg-2019-015215).
\end{abstract}

'Department of Interventional Neuroradiology, Fondation Ophtalmologique Adolphe de Rothschild, Paris, France ${ }^{2}$ Department of Neuroradiology, Centre Hospitalier Universitaire de Nancy, Nancy, France ${ }^{3}$ Department of

Biostatistics, EA 2694-Santé Publique,Epidémiologie et Qualité des Soins, CHU Lille, Lille, France

${ }^{4}$ Department of Stroke Center, Foch Hospital, Suresnes, France

\section{Correspondence to}

Dr Robert Fahed, Department of Interventional Neuroradiology, Fondation Ophtalmologique Adolphe de Rothschild, Paris 75019, France; robert.fahed@ hotmail.fr

Received 19 June 2019 Revised 13 July 2019 Accepted 17 July 2019 Published Online First 30 August 2019

\section{ABSTRACT}

Background The 'first pass effect' (FPE), which was originally described with stent retrievers, designates a (near-)complete revascularization obtained after a single device pass with no rescue therapy, and is associated with improved clinical outcome and decreased mortality. Objective We report the rate and benefits of FPE in the Aspiration versus Stent Retriever (ASTER) trial.

Materials and methods ASTER is a randomized trial comparing angiographic revascularization with the stent retriever (SR) and contact aspiration (CA) thrombectomy techniques, assessed by an external core laboratory using the modified Thrombolysis in Cerebral Infarction (mTICI) scale. Rates of FPE (defined by $\mathrm{mTICI} 2 \mathrm{c} / 3$ after a single pass with no rescue therapy) were compared between patients treated with SR and CA techniques. Outcomes were compared between FPE-SR and FPE-CA patients, and between FPE and non-FPE patients.

Results FPE was achieved in 97/336 patients (28.9\%), with no significant difference between SR and CA (respectively 53/169 patients (31.3\%) vs 44/167 patients (26.3\%), adjusted RR for CA versus SR $0.84,95 \% \mathrm{Cl}$ 0.54 to $1.31 ; p=0.44)$. After prespecified adjustment for allocated arm and randomization stratification factors, FPE in patients was associated with a significantly improved clinical outcome and a decreased mortality, and a significantly lower rate of hemorrhagic transformation and procedural complications than in non-FPE patients.

Conclusion In the ASTER trial, similar rates of FPE were achieved with SR and CA, and FPE was associated with a significantly improved outcome. New techniques and devices to improve the rate of FPE are warranted. Trial registration number Unique identifier: NCT02523261.

\section{INTRODUCTION}

Mechanical thrombectomy (MT) is now the standard of care for acute ischemic stroke with emergent large vessel occlusion, ${ }^{1}$ providing a high rate of successful revascularization (defined by a modified Thrombolysis in Cerebral Infarction (mTICI) of $2 b / 3)^{2}$ with excellent safety. ${ }^{3-5}$ It has recently been shown that better revascularization after MT is associated with a more favorable outcome. Notably, patients with mTICI $2 c / 3$ at the end of the procedure did significantly better than those with
mTICI $2 b .{ }^{6}$ Studies also suggest that multiple device passes are associated with a lower rate of both successful revascularization and favorable clinical outcome ${ }^{7}$ and higher rates of hemorrhagic transformations. ${ }^{8}$ A new index has thus been proposed: the 'first pass effect' (FPE), which symbolizes 'the ideal scenario' and is defined by a (near-)complete revascularization after a single pass of the device with no rescue therapy. ${ }^{9}{ }^{10}$ Zaidat et al have shown that patients with a FPE had a significantly improved outcome. However, this study included mostly patients treated with stent retrievers, and angiographic revascularization was not assessed by an independent core laboratory.

Using the data from the Aspiration versus Stent Retriever (ASTER) trial, which included randomized allocation of stent retriever (SR) or contact aspiration (CA) techniques, and external adjudication of angiographic outcome by an independent core laboratory, ${ }^{11} 12$ we aimed to study whether SR and CA achieved similar rates of FPE with similar outcomes, and, further, to assess the impact of FPE (with either SR or CA) on efficacy and safety outcomes after MT.

\section{METHODS}

Data were extracted from the ASTER trial, which was a randomized, multicenter, open-label, blinded endpoint clinical trial, designed to compare the effect of two first-line strategies for MT (SR vs CA) on reperfusion rates after completion of the endovascular procedures. ${ }^{1112}$ Institutional review board approval and written informed consent were obtained. The data supporting the findings of this study are available from the corresponding author on reasonable request.

\section{Patient population}

The ASTER trial enrolled adults admitted with suspected ischemic stroke secondary to occlusion of the anterior circulation within 6 hours of onset of symptoms. Patients were randomly assigned to first-line CA $(n=192)$ or first-line SR $(n=189)$ immediately before MT. An independent core laboratory, blinded to treatment allocation, assessed the angiographic revascularization status after each device pass, using the mTICI scale. Patients in the FPE group were thus defined according to the original article, ${ }^{9}$ as follows: (1) a (near-)complete 
revascularization of the large vessel occlusion and its downstream territory (mTICI $2 \mathrm{c} / 3)^{13}$ assessed by the core laboratory (2) after the first pass of the device (SR or CA), (3) without use of rescue therapy. For the CA technique, any withdrawal of the aspiration catheter (even partial) counted as a pass: if, after partial withdrawal of the aspiration catheter, free flow was restored in the aspiration tube without any visible clot, an attempt to readvance the aspiration catheter at the clot level was considered as a second pass in the ASTER trial.

\section{Outcomes}

Primary outcome for this post hoc analysis of the ASTER trial was favorable clinical outcome, defined as a mRS score at 90 days of 0 to 2 or equal to pre-stroke mRS score.

Secondary efficacy outcomes included excellent outcome (90-day mRS score of 0 to 1 or equal to pre-stroke mRS score), the degree of disability assessed by overall distribution of the 90-day mRS (shift analysis), 24-hour National Institutes of Health Stroke Scale (NIHSS) score change, and 90=day allcause mortality. Safety outcomes included hemorrhagic complications on imaging at $24 \pm 12$ hours (any intracranial hemorrhage $(\mathrm{ICH})$ ), parenchymal hematoma and symptomatic ICH (defined as any ICH visualized on follow-up imaging study and associated with a four-point or more worsening on the NIHSS or that resulted in death) and procedure-related adverse events (defined as arterial perforation, arterial dissection, embolization in a new territory, or subarachnoid hemorrhage).

\section{Statistical analysis}

Data analysis was performed on 336 patients who received firstline MT (CA or SR). Continuous variables were expressed as means (SD) for normal distribution or medians (IQR) otherwise. Categorical variables were expressed as numbers (percentage). Normality of distributions was assessed using histograms and the Shapiro-Wilk test. We compared the rate of FPE between first-line MT strategy (CA vs SR) using generalized estimating equation (GEE) models (Poisson distribution, log-link function) to take into account the center effect and include the randomization stratification factor (use of intravenous thrombolysis before MT) as covariate; adjusted risk ratios (RRs) were derived from GEE model as effect size using the CA group as reference.

Association of baseline characteristics and binary outcomes (favorable and excellent outcome, 90-day all-cause mortality, and procedural and hemorrhagic complications) with FPE were also assessed using GEE models (Poisson distribution, log-link function) to take into account the center effect, the allocated arm (first-line CA vs SR technique) and the use of intravenous thrombolysis before MT as prespecified covariates. Effect sizes (between FPE group differences) were derived from GEE models as adjusted RRs. Comparison of the overall distribution of mRS scores was performed using a mixed ordinal logistic regression model (shift analysis) including center as random effect, and FPE group, first-line MT strategy, and use of intravenous recombinant tissue plasminogen activator ( $\mathrm{rtPA}$ ) before $\mathrm{MT}$ as fixed effects; the common OR for a one-point improvement in mRS score was derived from this model as effect size using the non-FPE group as reference.

Comparison of 24-hour change in NIHSS score was performed using a linear mixed model including FPE group, admission NIHSS score, first-line MT strategy, and use of intravenous rtPA before MT as fixed effects and center as a random effect; adjusted between-group mean difference (FPE vs non-FPE group) was derived from this model as effect size. Normality of model residuals was checked and satisfied. Comparisons of outcomes were further adjusted for onset to groin puncture time and significant baseline between-group differences (namely, fully adjusted analyses), except the clot length which was unmeasurable in $22.6 \%$ of patients. We further investigated the association of FPE and outcomes according to first-line MT strategy by including the corresponding interaction term in previous regression models. Statistical testing was conducted at the twotailed level of $\mathrm{p}<0.05$. No adjustment for multiple testing was applied and thus results are considered exploratory. Data were analyzed using SAS software, version 9.3 (SAS Institute, Cary, North Carolina, USA).

\section{RESULTS}

\section{Patient population}

Between October 2015 and October 2016, a total of 381 patients were randomized in the ASTER trial. Of these, 45 were not treated by MT and were excluded from our study (figure 1).

Ninety-seven patients had (near-)complete reperfusion (mTICI $2 \mathrm{c} / 3$ ) after the first pass of the MT device (FPE, 28.9\%, 95\% CI $24.0 \%$ to $33.7 \%$ ). FPE was achieved in 53/169 patients treated with first-line SR (31.3\%) and 44/167 patients treated with first-line CA (26.3\%) (adjusted RR for CA vs SR, 0.84; 95\% CI 0.54 to $1.31 ; \mathrm{p}=0.44)$. As shown in table $1, \mathrm{FPE}$ was associated with pre-stroke $\mathrm{mRS}$ score $(\mathrm{RR}=1.64 ; 95 \% \mathrm{CI} 1.23$ to 2.17$)$ and higher Alberta Stroke Progam Early CT Score (ASPECTS) (RR per 1-point increase, 1.08; 95\% CI 1.01 to 1.15). We found no significant association between occlusion location or stroke etiology and FPE.

\section{Efficacy outcomes}

Favorable outcome was achieved in 56/93 FPE patients $(60.2 \%)$, with no significant difference between techniques: 28/51 patients with an FPE after SR (54.9\%) versus 28/42 patients with an FPE after CA (66.7\%), $\mathrm{p}=0.29$. Further analyses comparing outcomes between FPE and non-FPE patients according to firstline thrombectomy technique (SR or CA) are available in online supplementary table I. We found no significant heterogeneity in association of FPE with clinical outcomes across the first-line thrombectomy strategy (CA vs SR).

Table 2 shows results of a multivariate analysis comparing FPE and non-FPE patients. Favorable outcome was more often observed when FPE was achieved $(60.2 \%$ vs $43.7 \%$ in patients without FPE), with a prespecified adjusted RR associated with FPE of 1.38 (95\% CI 1.22 to 1.56). This difference was not modified after further adjustment on pre-stroke mRS score, ASPECTS, type of anesthesia, and onset to groin puncture time (table 2).

A similar effect of FPE was found when analyzing overall $\mathrm{mRS}$ distribution (figure 2, adjusted for prespecified factors $\mathrm{p}=0.041$ ); however, the association of overall $\mathrm{mRS}$ distribution and FPE did not remain significant in fully adjusted analysis, with a common OR for one-point mRS improvement of 1.44 (95\% CI 0.91 to 2.27 ). In addition, FPE was not significantly associated with a greater rate of excellent outcome (table 2). At 24 hours, FPE was associated with a significantly higher decrease in NIHSS score, with a fully adjusted mean difference of 3.5 points (95\% CI 1.5 to 5.6) in favor of the FPE group. 90-Day allcause mortality was significantly lower in cases of FPE $(12.9 \%$ vs $21.8 \%)$, with a fully adjusted RR of 0.56 (95\% CI 0.35 to 0.88 ).

\section{Safety outcomes}

Hemorrhagic transformation (of any type) and parenchymal hematoma (PH) occurred less frequently when FPE was achieved 


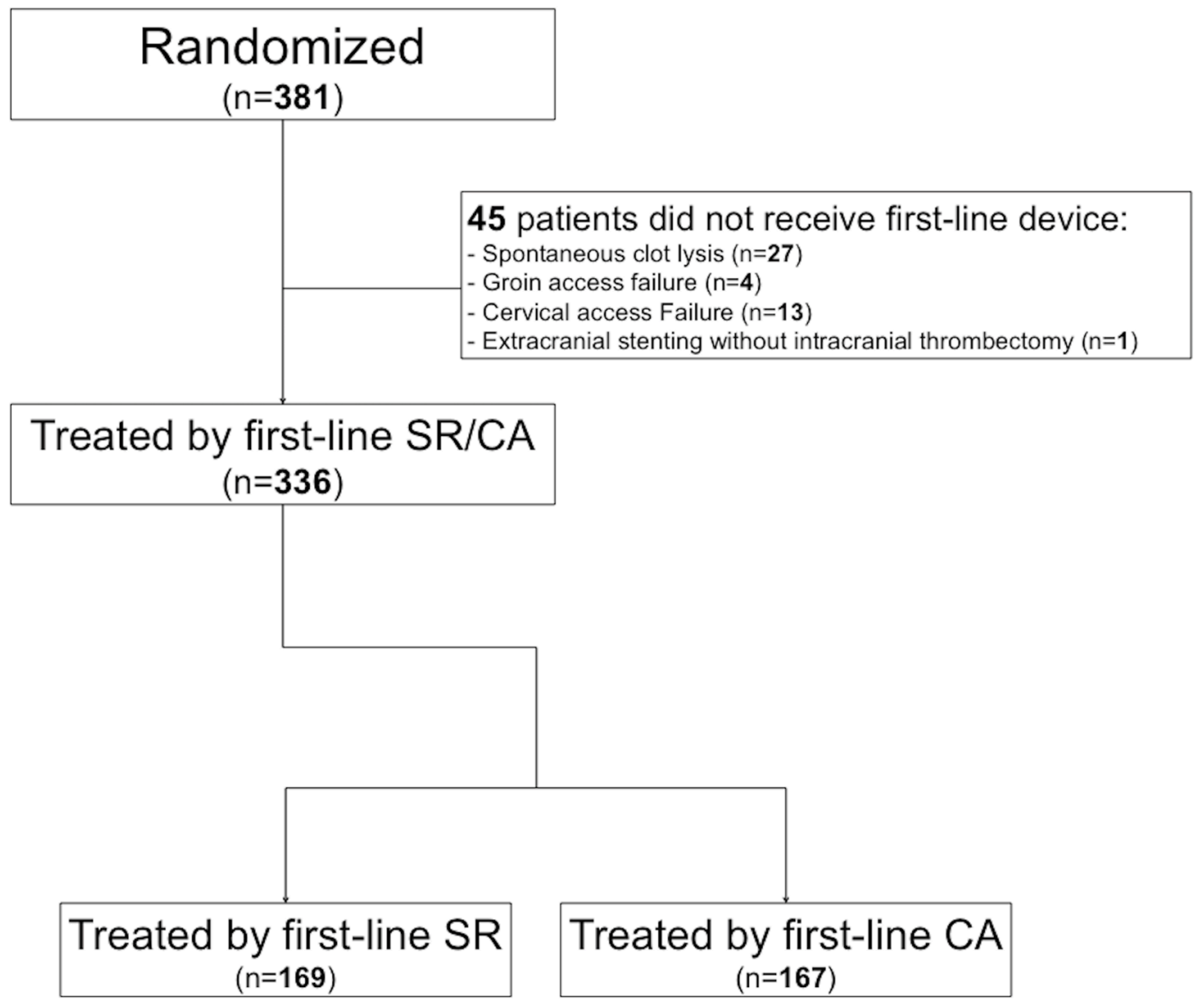

Figure 1 Study flow chart. CA, contact aspiration; SR, stent retriever

(table 2). In fully adjusted analyses, the difference remained significant, with an adjusted RR associated with FPE of 0.84 (95\% CI 0.71 to 1.00 ) for any intracranial hemorrhage and 0.28 (95\% CI 0.13 to 0.56 ) for PH. However, no difference in symptomatic intracranial hemorrhage was observed, with a rate of $5.2 \%$ in the FPE group and $6.0 \%$ in the non-FPE group. Procedure-related adverse events occurred less frequently in cases of FPE (10.3\% vs 20.5\%, prespecified adjusted RR, 0.51; $95 \%$ CI 0.26 to 0.99 ). In fully adjusted analysis, this difference was of borderline of significance $(p=0.067$, table 2$)$.

Although the heterogeneity test did not reach significance, in CA-treated patients, we found a significantly lower rate of hemorrhagic transformations (of any type) and procedurerelated adverse events in FPE than in non-FPE patients. No such differences were seen in SR-treated patients (online supplementary table I).

\section{DISCUSSION}

A first pass effect was achieved in $29 \%$ of patients in the ASTER trial, with no significant difference between stent retriever and contact aspiration techniques. In accordance with previous findings, ${ }^{9}$ FPE was associated with a higher rate of favorable outcome and a lower rate of mortality. In our study, FPE was also associated with a significantly reduced rate of hemorrhagic transformation and procedural complications.

MT has shown considerable benefits for acute ischemic stroke with emergent large vessel occlusion. ${ }^{3}$ Ideally, thrombectomy should provide a fast, ${ }^{14}$ complete, ${ }^{6}$ and safe ${ }^{15}$ revascularization. The FPE summarizes all these features and can be used for benchmarking of future thrombectomy devices and techniques.
In the ASTER trial, both SR and CA techniques achieved similar rates of FPE, with similar rates of favorable outcome.

Our study shows a similar rate of FPE to that of Zaidat et $a l^{9}(28.9 \%$ vs $25.1 \%)$ and confirms their findings of the clinical benefits of FPE on stroke outcome: we found that patients with FPE had a significantly higher rate of favorable clinical outcome but also a significantly lower rate of mortality. By definition, FPE is associated with a shorter procedural time, ${ }^{9}$ as no additional device passes or drugs are required to achieve complete revascularization. Consequently, the beneficial effects of FPE are obviously related to the speed of the procedure and the (near-) complete revascularization achieved. A recent meta-analysis has shown that the probability of successful revascularization decreases significantly with time between hospital arrival or first imaging to groin puncture. ${ }^{16}$ In order to verify that the effects of FPE obtained in our study were not simply the consequence of faster preprocedural management, we integrated time from onset to groin puncture in our multivariate analysis and showed that the beneficial effects of FPE persisted (table 2). A previous analysis of ASTER data also showed that there was no significant interaction between $\mathrm{mTICI} 2 \mathrm{c} / 3$ grade and onset-to-reperfusion time on favorable outcome. ${ }^{6}$ These results suggest that underlying reasons, other than just a faster and better revascularization, may explain the effects of FPE. A new finding in our study is the rate of hemorrhagic transformations (of any type or of PH-type only), which was also significantly lower in patients with FPE. This is in line with previous studies which suggested that occurrence of hemorrhagic transformation increases when the degree of revascularization decreases ${ }^{6}$ and when the number of device passes increases. ${ }^{8}$ 
Table 1 Baseline characteristics according to first pass effect in the ASTER trial

\begin{tabular}{|c|c|c|c|c|}
\hline Characteristics & FPE $(n=97)$ & Non-FPE $(n=239)$ & $P$ value & RR $(95 \% \mathrm{Cl})$ \\
\hline \multicolumn{5}{|l|}{ Demographics } \\
\hline Age, years, mean (SD) & $70.5(14.0)$ & $69.0(14.9)$ & 0.13 & $1.06(0.98 \text { to } 1.14)^{*}$ \\
\hline Men & 44/97 (45.4) & $130 / 239(54.4)$ & 0.18 & $0.76(0.50$ to 0.15$)$ \\
\hline \multicolumn{5}{|l|}{ Medical history } \\
\hline Hypertension & $53 / 95(55.8)$ & $145 / 235(61.7)$ & 0.12 & 0.84 (0.66 to 1.05$)$ \\
\hline Diabetes & 19/96 (19.8) & 49/234 (20.9) & 0.53 & 0.94 (0.77 to 1.15$)$ \\
\hline Hypercholesterolemia & $34 / 96(35.4)$ & $86 / 232(37.1)$ & 0.68 & $0.95(0.75$ to 1.20$)$ \\
\hline Current smoking & 16/76 (21.1) & $41 / 206(19.9)$ & 0.77 & 1.07 (0.68 to 1.67$)$ \\
\hline Coronary artery disease & 14/95 (14.7) & 45/232 (19.4) & 0.10 & 0.77 (0.56 to 1.06$)$ \\
\hline Previous stroke or TIA & $20 / 96(20.8)$ & 39/236 (16.5) & 0.19 & $1.23(0.90$ to 1.70$)$ \\
\hline Previous antithrombotic medications & $43 / 93(46.2)$ & $118 / 237(49.8)$ & 0.28 & $0.89(0.72$ to 1.10$)$ \\
\hline Antiplatelet & 28/93 (30.1) & $76 / 237(32.1)$ & 0.50 & 0.93 (0.75 to 1.15$)$ \\
\hline Anticoagulant & 15/93 (16.1) & $50 / 237(21.1)$ & 0.083 & 0.75 (0.53 to 1.04$)$ \\
\hline \multicolumn{5}{|l|}{ Current stroke event } \\
\hline Systolic blood pressure, $\mathrm{mm} \mathrm{Hg}$, mean (SD)† & $148(25)$ & $146(26)$ & 0.26 & $1.03(0.98 \text { to } 1.08)^{*}$ \\
\hline NIHSS score, mean (SD)‡ & $15.8(6.5)$ & $16.4(6.0)$ & 0.27 & $0.94(0.84$ to 1.05$) \S$ \\
\hline Pre-stroke mRS score $\geq 1$ & $24 / 96(25.0)$ & $33 / 239(13.8)$ & $<0.001$ & 1.64 (1.23 to 2.17$)$ \\
\hline ASPECTS, median (IQR)ף & $8(6$ to 9$)$ & 7 (5 to 9$)$ & 0.014 & $1.08(1.01 \text { to } 1.15)^{* *}$ \\
\hline \multicolumn{5}{|l|}{ Site of occlusion } \\
\hline M1-MCA & $61 / 97(62.9)$ & $134 / 239(56.1)$ & 0.31 & 1.00 (reference) \\
\hline M2-MCA & 23/97 (23.7) & $53 / 239(22.2)$ & & 1.00 (0.61 to 1.62$)$ \\
\hline Intracranial ICA or tandem & 13/97 (13.4) & $52 / 239(21.8)$ & & $0.63(0.34$ to 1.16$)$ \\
\hline Favorable collaterals & 23/81 (28.4) & 43/184 (23.4) & 0.40 & 1.20 (0.78 to 1.84$)$ \\
\hline Clot length, mm, median (IQR)†† & 11 (8 to 16) & 13 (9 to 20$)$ & 0.098 & $0.97(0.94 \text { to } 1.01)^{* *}$ \\
\hline Clot burden, median (IQR)‡‡ & 6 (4 to 8$)$ & 7 (5 to 8$)$ & 0.18 & $0.97(0.93 \text { to } 1.02)^{* *}$ \\
\hline \multicolumn{5}{|l|}{ Suspected stroke cause } \\
\hline Large artery atherosclerosis & 10/97 (10.3) & $18 / 239(7.5)$ & 0.15 & 1.00 (reference) \\
\hline Cardioembolic & $33 / 97(34.0)$ & $109 / 239(45.6)$ & & $0.66(0.41$ to 1.05$)$ \\
\hline Other or unknown & $54 / 97(55.7)$ & $112 / 239(46.9)$ & & 0.91 (0.65 to 1.27 ) \\
\hline Intravenous rtPA & $61 / 97(62.9)$ & $155 / 239(64.9)$ & 0.51 & $0.94(0.78$ to 1.13$)$ \\
\hline \multicolumn{5}{|l|}{ Endovascular treatment } \\
\hline General anesthesia & $7 / 97(7.2)$ & $38 / 239(15.9)$ & 0.077 & $0.50(0.23$ to 1.08$)$ \\
\hline Onset to groin puncture time, min, median (IQR)‡ & 230 (179 to 283$)$ & 230 (184 to 279$)$ & 0.34 & 1.02 (0.97 to 1.07$) \S \S$ \\
\hline Onset to imaging & 108 (80 to 149$)$ & 114 (84 to 150$)$ & 0.34 & $1.02(0.97$ to 1.07$) \S \S$ \\
\hline Imaging to groin puncture & $112(69$ to 154$)$ & 110 (62 to 150$)$ & 0.93 & $1.00(0.90$ to 1.12$) \S \S$ \\
\hline
\end{tabular}

Values expressed as no/total no (\%) unless otherwise indicated.

${ }^{*} O R$ calculated per 10 unit increase.

†Nine missing values.

¥Three missing values.

$\S 0 R$ calculated per 30 unit increase.

IFour missing values.

${ }^{* *}$ OR calculated per 1 unit increase.

t†Seventy-six6 missing values.

$\ddagger \neq$ One hundred and five missing values.

$\S \S 0 R$ calculated per 30 unit increase.

ASPECTS, Alberta Stroke Programme Early CT score; FPE, first pass effect; ICA, internal carotid artery; MCA, middle cerebral artery; mRS, modified Rankin Scale, NIHSS, National Institutes of Health Stroke Scale; rtPA, recombinant tissue plasminogen activator; TIA, transient ischemic attack.

We also found significantly fewer procedure-related adverse events in patients with FPE. Because the risk of complications is inherently linked to the number of intracranial maneuvers, one would expect a single pass to be safer than multiple passes. Moreover, studies suggest that multiple device passes (both SR and CA) can cause local endothelial lesions, ${ }^{717} 18$ which might promote local re-occlusion.

The design of the ASTER trial and the relatively small population does not allow us to rigorously identify predictors of FPE, therefore the analyses comparing baseline characteristics 
Table 2 Comparison of efficacy and safety outcomes according to first pass effect in the ASTER trial

\begin{tabular}{|c|c|c|c|c|c|c|}
\hline Outcomes & $\begin{array}{l}\text { FPE } \\
(n=97)\end{array}$ & $\begin{array}{l}\text { Non-FPE } \\
(n=239)\end{array}$ & $\operatorname{RR}(95 \% \mathrm{Cl})^{*}$ & $P$ value* & RR $(95 \% \mathrm{Cl}) \dagger$ & $P$ valuet \\
\hline \multicolumn{7}{|l|}{ Efficacy outcomes } \\
\hline Favorable outcome & $56 / 93(60.2)$ & $100 / 229(43.7)$ & 1.38 (1.22 to 1.56$)$ & $<0.001$ & 1.31 (1.18 to 1.45$)$ & $<0.001$ \\
\hline Excellent outcome & $41 / 93(44.1)$ & $84 / 229(36.7)$ & $1.18(0.94$ to 1.49$)$ & 0.14 & 1.11 (0.90 to 1.38$)$ & 0.32 \\
\hline 90-Day mortality & 12/93 (12.9) & $50 / 229(21.8)$ & 0.58 (0.37 to 0.92$)$ & 0.019 & $0.56(0.35$ to 0.88$)$ & 0.011 \\
\hline Any ICH & 38/96 (39.6) & $120 / 234(51.3)$ & 0.77 (0.66 to 0.90$)$ & $<0.001$ & $0.84(0.71$ to 1.00$)$ & 0.044 \\
\hline Parenchymal hematoma & $7 / 96(7.3)$ & 45/234 (19.2) & $0.38(0.22$ to 0.64$)$ & $<0.001$ & $0.28(0.13$ to 0.56$)$ & $<0.001$ \\
\hline $\mathrm{sICH}$ & $5 / 96(5.2)$ & $14 / 234(6.0)$ & 0.87 (0.39 to 1.92$)$ & 0.74 & 0.82 (0.27 to 2.46$)$ & 0.72 \\
\hline Procedural complications & 10/97 (10.3) & 49/239 (20.5) & 0.51 (0.26 to 0.99$)$ & 0.046 & $0.58(0.32$ to 1.04$)$ & 0.067 \\
\hline
\end{tabular}

Values expressed as no/total no (\%), unless otherwise stated

* Calculated using the non-FPE group as reference, after prespecified adjustment for center, first-line MT strategy and intravenous rtPA.

tCalculated using the non-FPE group as reference after adjustment for center, first-line MT strategy, intravenous rtPA, significant baseline differences (except clot length) and onset to groin puncture time.

¥Mean change $(95 \% \mathrm{Cl})$ adjusted on baseline NIHSS score, center, first-line MT strategy and intravenous rtPA.

$\S$ Adjusted mean difference (FPE vs non-FPE).

FPE, first pass effect; ICH, intracranial hemorrhage; MT, mechanical thrombectomy; NIHSS, National Institutes of Health Stroke Scale; RR, risk ratio; rtPA, recombinant tissue plasminogen activator; sICH, symptomatic intracranial hemorrhage.

of FPE and non-FPE patients (table 1) should be interpreted with caution. However, the use of the ASTER cohort for the study of FPE presents two major strengths. First, the randomized allocation of treatment strategy (SR and CA) allows a rigorous comparison of the two techniques and suggests here that both achieve similar rates of FPE. Second, the external adjudication of angiographic outcome ensures a strong and homogeneous assessment of mTICI $2 \mathrm{c} / 3$ outcome, as previous studies suggest that investigator-assessed angiographic outcomes may be inconsistent. ${ }^{19} 20$

Future trials should now focus on technical refinements to increase the rate of FPE. Studies suggest that the size of the stent retriever ${ }^{21}$ and the duration of its deployment ${ }^{22}$ might influence the rate of complete revascularization after one pass. For the CA technique, the size of the aspiration catheter might also be a critical factor. ${ }^{23-26}$ The choice of the middle cerebral artery branch for device placement may also play an important role. ${ }^{27}$ Zaidat et al suggest that the use of a balloon-guide catheter strongly increases the rate of FPE. ${ }^{9}$ The use of a combined approach $(\mathrm{CA}+\mathrm{SR})$ might also increase the rate of FPE. Multiple $\mathrm{SR}+\mathrm{CA}$ techniques have been described in the literature. ${ }^{28-30}$ The results of the ASTER-2 trial (NCT03290885), comparing SR with SR+CA, should provide more insight into the benefits of this technique.

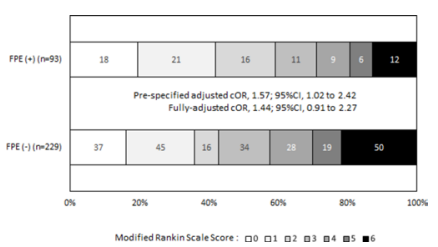

Figure 2 Distribution of modified Rankin Scale scores at 90 Days according to first pass effect in the ASTER trial. COR was calculated for one-point improvement in the modified Rankin Scale score using a mixed ordinal logistic regression model including center as random effect and first-line mechanical thrombectomy strategy and intravenous recombinant tissue plasminogen activator as fixed effects. COR, common odds ratio; FPE, first pass effect.
Our study has several limitations. The ASTER trial was not originally designed to compare mTICI $2 \mathrm{c} / 3$ rates after a single pass between SR and CA, but mTICI $2 b / 3$ rates at the end of the procedures; therefore, it may be underpowered to detect a difference between the two techniques for achieving FPE. A superiority trial that would try to show a $10 \%$ superiority of one technique over the other for FPE rates (35\% vs $25 \%)$ with a statistical power of $90 \%$ and a two-sided $\alpha$ error of 0.05 would require at least 878 patients.

\section{CONCLUSION}

A first pass effect was achieved in 29\% of patients in the ASTER trial, with no significant difference between contact aspiration and stent retriever techniques. Patients achieving FPE had a significantly better clinical outcome and a lower mortality. Further studies are necessary to determine the predictors of FPE and to develop methods to increase the rate of complete revascularization after a single pass.

Collaborators ASTER trial investigators: Hocine Redjem, Gabriele Ciccio, Stanislas Smajda, Mikael Mazighi, Jean-Philippe Desilles, Simon Escalard, Georges Rodesch, Arturo Consoli, Oguzhan Coskun, Federico Di Maria, Frédéric Bourdain, Jean Pierre Decroix, Adrien Wang, Maya Tchikviladze, Serge Evrard, Francis Turjman, Benjamin Gory, Paul Emile Labeyrie, Roberto Riva, Charbel Mounayer, Suzanna Saleme, Vincent Costalat, Alain Bonafe, Omer Eker, Grégory Gascou, Cyril Dargazanli, Serge Bracard, Romain Tonnelet, Anne Laure Derelle, René Anxionnat, Hubert Desal, Romain Bourcier, Benjamin Daumas-Duport, Jérome Berge, Xavier Barreau, Gauthier Marnat, Lynda Djemmane, Julien Labreuche, Alain Duhamel

Contributors Study design: CD, MP, JL, BL, RF. Acquisition, analysis, or interpretation of data: all authors. Drafting of the manuscript: $C D, M P, J L, B L, R F$. Statistical analysis: JL. Supervision: RF.

Funding The authors have not declared a specific grant for this research from any funding agency in the public, commercial or not-for-profit sectors.

Competing interests None declared.

Patient consent for publication Not required.

Provenance and peer review Not commissioned; externally peer reviewed.

Data sharing statement Data, analytic methods, and study materials will be made available to any researcher for purposes of reproducing the results or 
replicating the procedure. Requests to receive these materials should be sent to the corresponding author, who will maintain their availability.

Open access This is an open access article distributed in accordance with the Creative Commons Attribution Non Commercial (CC BY-NC 4.0) license, which permits others to distribute, remix, adapt, build upon this work non-commercially, and license their derivative works on different terms, provided the original work is properly cited, appropriate credit is given, any changes made indicated, and the use is non-commercial. See: http://creativecommons.org/licenses/by-nc/4.0/.

ORCID iD

Robert Fahed http://orcid.org/0000-0002-1887-5097

\section{REFERENCES}

1 Leslie-Mazwi T, Chandra RV, Baxter BW, et al. ELVO: an operational definition. J Neurointerv Surg 2018;10:507-9.

2 Zaidat 00, Yoo AJ, Khatri P, et al. Recommendations on angiographic revascularization grading standards for acute ischemic stroke: a consensus statement. Stroke 2013:44:2650-63.

3 Powers WJ, Rabinstein AA, Ackerson T, et al. 2018 Guidelines for the early management of patients with acute ischemic stroke: a guideline for healthcare professionals from the American Heart Association/American Stroke Association. Stroke 2018;49:e46-110

4 Leslie-Mazwi T, Chandra RV, Fraser JF, et al. AHA/ASA 2018 AIS guidelines: impact and opportunity for endovascular stroke care. J Neurointerv Surg 2018;10:817-7.

5 Mocco J, Fiorella D, Fargen KM, et al. Endovascular therapy for acute ischemic stroke is indicated and evidence based: a position statement. J Neurointerv Surg 2015:7:79-81.

6 Dargazanli C, Fahed R, Blanc R, et al. Modified thrombolysis in cerebral infarction 2cl thrombolysis in cerebral infarction 3 reperfusion should be the aim of mechanical thrombectomy: insights from the ASTER trial (Contact Aspiration Versus Stent Retriever for Successful Revascularization). Stroke 2018:49:1189-96.

7 Flottmann $\mathrm{F}$, Leischner $\mathrm{H}$, Broocks $\mathrm{G}$, et al. Recanalization rate per retrieval attempt in mechanical thrombectomy for acute ischemic stroke. Stroke 2018;49:2523-5.

8 Bourcier R, Saleme S, Labreuche J, et al. More than three passes of stent retriever is an independent predictor of parenchymal hematoma in acute ischemic stroke. J Neurointerv Surg 2019:11:625-9.

9 Zaidat 00, Castonguay AC, Linfante I, et al. First pass effect. Stroke 2018;49:660-6.

10 Jindal G, Carvalho HP, Wessell A, et al. Beyond the first pass: revascularization remains critical in stroke thrombectomy. J Neurointerv Surg 2019:doi: 10.1136/ neurintsurg-2019-014773 [Epub ahead of print].

11 Lapergue B, Blanc R, Gory B, et al. Effect of endovascular contact aspiration vs stent retriever on revascularization in patients with acute ischemic stroke and large vessel occlusion: the ASTER randomized clinical trial. JAMA 2017:318:443-52.

12 Lapergue B, Labreuche J, Blanc R, et al. First-line use of contact aspiration for thrombectomy versus a stent retriever for recanalization in acute cerebral infarction: the randomized ASTER study protocol. Int J Stroke 2018;13:87-95.

13 Liebeskind DS, Bracard S, Guillemin F, et al. eTICI reperfusion: defining success in endovascular stroke therapy. J Neurointerv Surg 2019;11:433-8.
14 Saver JL, Goyal M, van der Lugt A, et al. Time to treatment with endovascular thrombectomy and outcomes from ischemic stroke: a meta-analysis. JAMA 2016;316:1279-88.

15 Balami JS, White PM, McMeekin PJ, et al. Complications of endovascular treatment for acute ischemic stroke: prevention and management. Int J Stroke 2018;13:348-61.

16 Bourcier R, Goyal M, Liebeskind DS, et al. Association of time from stroke onset to groin puncture with quality of reperfusion after mechanical thrombectomy: a metaanalysis of individual patient data from 7 randomized clinical trials. JAMA Neurol 2019 [Epub ahead of print $22 \operatorname{Jan} 2019$ ].

17 Peschillo S, Diana F, Berge J, et al. A comparison of acute vascular damage caused by ADAPT versus a stent retriever device after thrombectomy in acute ischemic stroke: a histological and ultrastructural study in an animal model. J Neurointerv Surg 2017:9:743-9

18 Arai $\mathrm{D}$, Ishii $\mathrm{A}$, Chihara $\mathrm{H}$, et al. Histological examination of vascular damage caused by stent retriever thrombectomy devices. J Neurointerv Surg 2016;8:992-5.

19 Fahed R, Ben Maacha M, Ducroux C, et al. Agreement between core laboratory and study investigators for imaging scores in a thrombectomy trial. J Neurointerv Surg 2018:10:e30.

20 Zhang G, Treurniet KM, Jansen IGH, et al. Operator versus core lab adjudication of reperfusion after endovascular treatment of acute ischemic stroke. Stroke 2018;49:2376-82.

21 Zaidat 00 , Haussen DC, Hassan AE, et al. Impact of stent retriever size on clinical and angiographic outcomes in the STRATIS stroke thrombectomy registry. Stroke 2019:50:441-7.

22 Kannath SK, Rajan JE, Sylaja PN, et al. Dwell time of stentriever influences complete revascularization and first-pass $\mathrm{TICl} 3$ revascularization in acute large vessel occlusive stroke. World Neurosurg 2018;110:169-73.

23 Alawieh A, Chatterjee AR, Vargas J, et al. Lessons learned over more than 500 stroke thrombectomies using ADAPT with increasing aspiration catheter size. Neurosurgery 2018 [Epub ahead of print 10 Nov 2018].

24 Anadani M, Alawieh A, Vargas J, et al. First attempt recanalization with ADAPT: rate, predictors, and outcome. J Neurointerv Surg 2019;11:641-5.

25 Blanc R, Redjem H, Ciccio G, et al. Predictors of the aspiration component success of a direct aspiration first pass technique (ADAPT) for the endovascular treatment of stroke reperfusion strategy in anterior circulation acute stroke. Stroke 2017;48:1588-93.

26 Delgado Almandoz JE, Kayan Y, Wallace AN, et al. Larger ACE 68 aspiration catheter increases first-pass efficacy of ADAPT technique. J Neurointerv Surg 2019;11:141-6.

27 Maus V, Brehm A, Tsogkas I, et al. Stent retriever placement in embolectomy: the choice of the post-bifurcational trunk influences the first-pass reperfusion result in $\mathrm{M} 1$ occlusions. J Neurointerv Surg 2019;11:237-40.

28 Massari F, Henninger N, Lozano JD, et al. ARTS (Aspiration-Retriever Technique for Stroke): initial clinical experience. Interv Neuroradiol 2016:22:325-32.

29 Maus V, Behme D, Kabbasch C, et al. Maximizing first-pass complete reperfusion with SAVE. Clin Neuroradiol 2018;28:327-38.

30 McTaggart RA, Tung EL, Yaghi S, et al. Continuous aspiration prior to intracranial vascular embolectomy (CAPTIVE): a technique which improves outcomes. $J$ Neurointerv Surg 2017;9:1154-9. 